A. U. Anka ${ }^{1}$, I. N. Abdullahi ${ }^{1}$, K. Umar ${ }^{1}$, Z. M. Bello ${ }^{1}$, M. Mohammed ${ }^{2}$, S. Gachpaz

SARKhiz ${ }^{3}$, N. K. AbUbakar ${ }^{4}$, M. Alsabbagh ${ }^{5}$, A. N. Kamali ${ }^{6}$, G. Azizi ${ }^{7}$

\title{
Biological and clinical significance of T helper 17 cell deficiency: insight into monogenic defects
}

\author{
${ }^{1}$ Department of Medical Laboratory Science, College of Medical Sciences, Ahmadu Bello University, Zaria, Nigeria \\ ${ }^{2}$ Immunology Unit, Department of Medicine, Ahmadu Bello University, Zaria, Nigeria \\ ${ }^{3}$ Faculty of Medicine, Selcuk University, Konya, Turkey \\ ${ }^{4}$ Department of Health Services, Federal University Birnin Kebbi, Nigeria \\ ${ }^{5}$ Division of Translational Medicine, Research Branch, Sidra Medicine, Doha, Qatar \\ ${ }^{6}$ CinnaGen Medical Biotechnology Research Center, Alborz University of Medical Sciences, Karaj, Iran \\ ${ }^{7}$ Non-communicable Diseases Research Center, Alborz University of Medical Sciences, Karaj, Iran
}

\section{KEY WORDS}

Th17 cells; STAT1; STAT3; DOCK8; AIRE;

IL-17RA; chronic mucocutaneous candidiasis.

\section{Corresponding author}

Gholamreza Azizi

Non-communicable Diseases Research Center

Vice Chancellor for Research

Alborz University of Medical Sciences

Karaj, Iran

E-mail: azizi@abzums.ac.ir

Doi

10.23822/EurAnnACI.1764-1489.160

\begin{abstract}
Summary
Thelper 17 (Th17) are a $C D 4^{+}$T subpopulation cells which are involved in the host protection against microbes such as extracellular and intracellular bacteria, parasites, fungi, and viruses. Monogenic defects including those mutations in some genes such as the signal transducer and activator of transcription (STAT) 1 and 3, dedicator of cytokinesis 8 (DOCK8), autoimmune regulator (AIRE), and interleukin 17 receptor A (IL-17RA) can lead to impairment in Th17 cell development and function along with the concomitant increased risk for chronic mucocutaneous candidiasis (CMC). The immunologic abnormalities in these patients include low frequency of Th17 cells; defective cutaneous or in vitro T cell response to Candida species, andlor autoantibodies against relevant cytokines. This review outlines the biological characteristics and functionality of Th17 cells, as well as the clinical features of individuals with genetic defects associated with Th17 deficiency.
\end{abstract}

\section{Introduction}

$\mathrm{T}$ helper (Th) cells that synthesis Interleukin-17 (IL-17) are derived from $\mathrm{CD}^{+} \mathrm{T}$ cells subpopulation which are associated with protection of host against microbes including extracellular bacteria and quite a number of fungal agents (1). Disease progression in various autoimmunity and inflammatory disorders is due to direct involvement of Th17 cells which secret the IL17 family, cytokines including IL-17A and IL-17F, along with IL-22 and granulocyte- macrophage colony-stimulating factor (GM-CSF) (1). Cytokines like IL-17A and IL-17F often induce neutrophil production and local recruitment by controlling the expression of granulocyte-colony-stimulating factor (G-CSF) and tissue expression of CXCR2 ligands such as CXCL8 (IL-8), respectively (1). Neutrophils play a crucial role in the preven- tion of invasive fungal infections such as those caused by Candida $(2,3)$. Notably, Th17 cells analysis in human subjects and various murine models studies have confirmed that Th17 cells also play a pivotal function in mucosal immunity $(1,4)$.

Investigation on individuals that are prone to chronic mucocutaneous candidiasis (CMC) with defects in Th17 development and functionality due to single nucleotide polymorphism or as segment of syndrome have elucidated the involvement of Th17 cells in the protection of human host $(5,6)$. The processes of Th17 cell differentiation and inborn errors of Th17 cell function in affected patients have drawn attention in recent time (7). For instance, it has been reported that mutation in some genes such as DOCK8, STAT3, STAT1, AIRE, IL-17RA, IL-17F led to impairment of Th17 development and function in humans (8). 
In this review, we summarized the biological characteristics and functions of Th17 cells as well as the clinical features of patients with genetic deficiencies associated with Th17 deficiency.

\section{Biological characteristics and functions of Th17 cells}

Th17 cells are described as producers of TNF- $\alpha$ and IL17, which represents a group of cytokines that are made up of IL17A-F, IL6, 21, 22, and 23 (9). Investigators revealed Th17 cells as one of the T-helper cells progeny having a distinctive host defense function against different extracellular infectious agents via effectors mediation of its secreted cytokines (figure 1). However,

Figure 1 - The biological functions of effector Th17 cells in human.

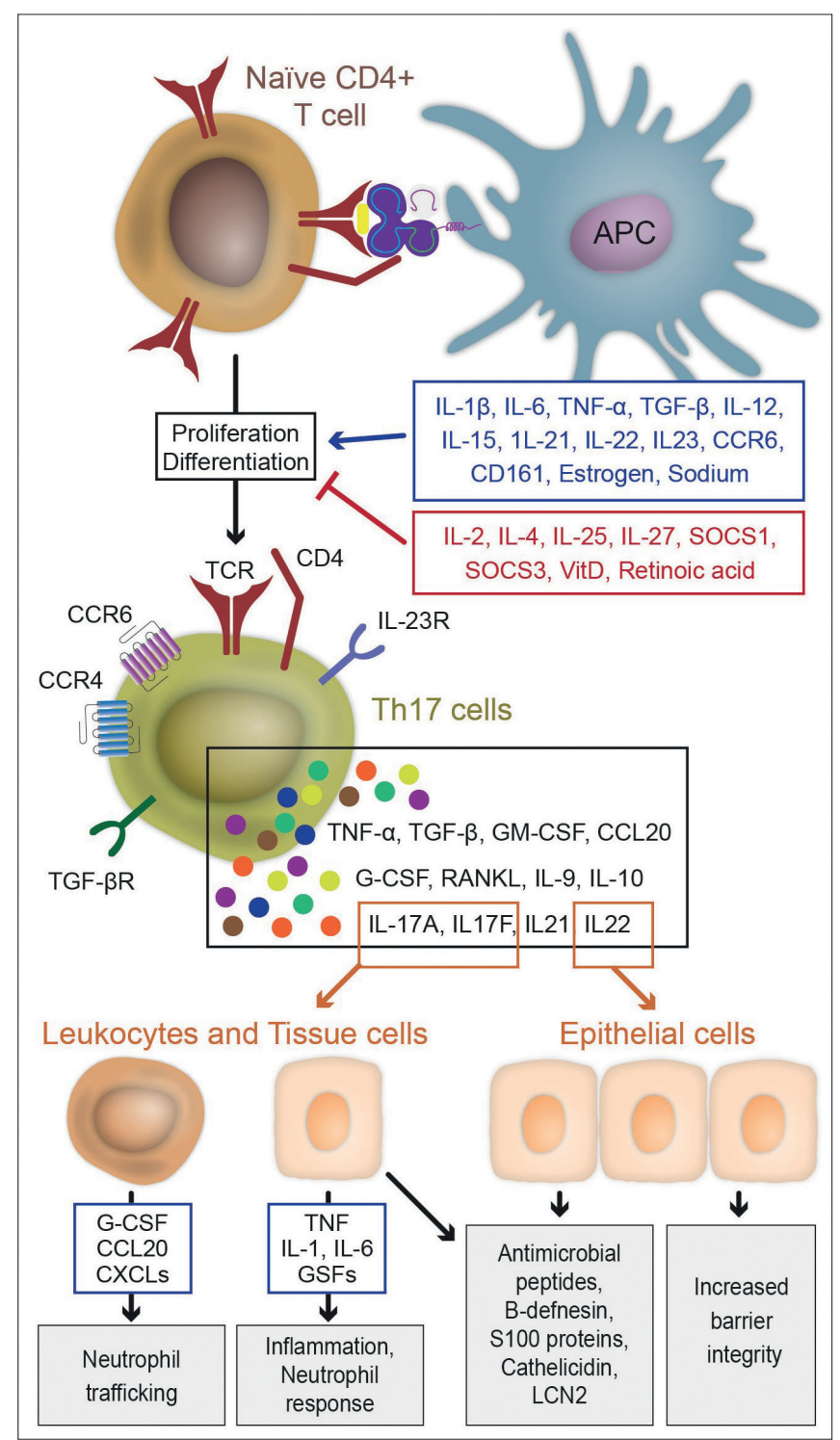

Th17 cells have been shown to be related with autoimmunity, carcinogenesis and certain chronic inflammatory diseases (10). Th17 cells and their associated cytokine IL-17 are responsible for various diseases such as psoriasis, asthma, tuberculosis and $\mathrm{CMCC}$ among many, but their precise roles in the pathogenesis of such diseases is not clearly understood. It was understood that IL-17 are associated with host defense against chronic inflammation, which also causes tissue damage or autoimmunity the activation of IL-17 signaling is via the IL-17 receptor binding by the IL-17 molecule, which in turn induces pro-inflammatory cytokines, neutrophil chemokines and antimicrobial peptides that are essential for antifungal activities (2). Since IL17 establishes a link between innate and adaptive immunity during the course of disease, it may have both physiological and pathological roles on host immune system. Evidence supports the notion that the dysregulated synthesis of IL17 and 21 by Th17 cells may contribute to the immunopathogenesis of autoimmune disorders (9).

\section{Intracellular Bacteria}

It has been studied that Th17 plays role in the immunogenicity of Mycobacterium tuberculosis but the precise role of its associated cytokine IL-17 in the infections is not clearly understood (11). In the early immune response to $M$. tuberculosis infection, Th17 cells stimulate recruitment of neutrophils, macrophages, and Th1 cells into the zone of inflammation while regulating the infection process as well (11). Th17 is also critical in the regulation of Th1 responses to $M$. tuberculosis infection as well (11). Nonetheless, insufficient Th17 cytokines appears unessential for protection against mycobacteria, and also in the lung, IL-17-producing cells were identified before the recruitment of IFN- $\gamma$-producing cells following antigen vaccination (11). Interestingly, for the protective recall response, IL-23 is required instead of IL-12 via the production of granulomas and initiation of activated CD4 T cells, which is as a result of IL-17 induced chemokine expression of CXCL19, CXCL10, and CXCL11, the recruitment of nuetrophils that facilitate the formation of granulomasis induced by CXCR3 ligand $(11,12)$. Consequently, humans that have been previously exposed to $M$. tuberculosis possessed IL-17- and IL-22-producing cells in peripheral blood mononuclear cells (PBMCs) that have been sensitized with $M$. tuberculosis antigens (Mtb-Ags). Nonetheless IL-17 and IL-22 are produced by the same Th17 cells in mice $(13,14)$. The search for IL-17 and IL-22 double producer cells was never a success in humans. But T-cells responsible for the production of IL-22 were frequently seen than those that produced IL-17 (15). Hence, these findings indicate that more studies should be conducted to reveal the precise mechanisms of actions of Th17/ IL-17 in various $M$. tuberculosis strain infections or vaccinations. 


\section{Extracellular Bacteria}

Host immune response due to Th17 cells in countering the pathogenic effect of Pseudomonas aeruginosa, Klebsiella pneumoniae, Mycoplasma pneumoniae, Helicobacter pylori, and Citrobacter rodentium, has been investigated in different studies (16). In a research conducted by Aujla and colleagues highlighted some key findings on the IL-22 role in $K$. pneumoniae infection protection (17). Extracted tissue of $K$. pneumoniae infected mice demonstrated an IL-23 dependent IL-22 with relatively same kinetics as IL-17 and IL-17F (17).

The impact of both IL-17 and IL-22 in human bronchial epithelial (HBE) cells were revealed as well as off regulation of host defense genes (17) and that of IL-22 in epithelial cells regeneration post trauma (17).

The involvement of Th17 cells in patients infected with Helicobacter pylori (H. pylori) was also studied (17). A controlled analysis between the un-infected and infected biopsies of $H$. pylori revealed an increased concentration of IL-17 and IL-23 mRNA in the later. An increased in IL-17 was detected in both and $\mathrm{CD}^{+}$cells and freshly obtained gastric lamina propria mononuclear cells (GLPMC), while only $\mathrm{CD}^{+}$cells increase was detected in (GLPMC) and there is also increased levels of IFN- $\gamma$ expression due to IL-23 on specific CD4 T cells (18).

\section{Viruses}

In an effort to delineate the role of IL-17 in viral pathogenesis, over-expression of IL-17 was identified as a factor $(19,20)$, while the research conducted by Smiley and his colleagues also demonstrated that a successfully immunized mice with rotavirus vaccine shows the production of protective IF- $\gamma$ and IL-17 by Ag-specific CD4 T cells (21) despite the fact there is evidence of role of other factors in the protection against rotavirus because both IFN $\gamma-\mathrm{R}$ and IL-17R remain protected after successful immunization of KO mice (21).

Recently, an expression level of IL-17 and IL-22 was studied in HIV patient were increase concentration of IL- $17^{+} \mathrm{CD} 3^{+} \mathrm{CD} 4^{+}$ and $\mathrm{IL}-17^{+} \mathrm{CD}^{+} \mathrm{CD} 4 \mathrm{~T}$ cells peripheral blood in relation to HIV uninfected persons (22) while IL-22 and acute phase proteins high levels was seen in the studies by Misse et al., due to activated T-cells in HIV infected and uninfected individuals (23).

\section{Parasites}

In an effort to study the role of $\mathrm{Th} 17$ in parasitic infection, mice with IL-17R deficiency and Toxoplasma gondii infected have shown relatively higher mortality, as there is low neutrophil recruitment in different tissues and CXCL8 expression, which resulted in higher parasitic burden (24). There is an increase in Th17 cells due to immunization with schistisome egg antigen in CFA (25) also IL-23p19-/- with severely reduced immunopathology, associated with reduced IL-17 producing T-cells in granulomas and a diminishing neutrophil recruitment due to reduced chemokine levels (26). To further explore the role of Th17 cells in parasitic immune response different experimental models need to be studied.

\section{Fungi}

Protective functionalities of Th17 in fungal immunity have been demonstrated in different instances. It was found that Pneumocystis carinii express IL-1 $\beta$, IL-6 and IL-23. Inducing Th17 differentiation while blocking IL-23 or IL-17 by neutralizing antibodies significantly elevated the burden of Pneumocystic pneumonia. In addition, IL-23-deficient mice showed higher susceptibility to systemic Cryptococcus neoformans and pulmonary $P$. carinii infections $(18,19)$. Albicans candidiasis has also been shown to activate IL-23 expression in humans by memory $\mathrm{T}$ cells and monocyte-derived dendritic cells (DCs). These cells express CCR6 and CCR 4 to produce IL-17 (20). Although, the IL-23/IL-17 pathway has been shown to enhance inflammation that inhibits the protective Th1 response against Candida and Aspergillus species $(21,22)$, Th17 cytokines and Th17 cells have been implicated in immunity against several infectious agents. For now, the origin of these cytokines in the innate host defenses is not fully understood. Therefore, there is the need for further investigations into the role of Th17 cells in chronic infection or memory responses in order to fully understand the innate host defenses against fungi.

\section{Development and plasticity of Th17 cells}

In 2008 Cosmi et al. showed that all Th17 cells originate from $\mathrm{CD}_{161}{ }^{+}$naive $\mathrm{CD}^{+}{ }^{+} \mathrm{T}$ cells and introduced $\mathrm{CD} 161$ as a surface marker for human Th17 cells (23). Studies by Bettelli et al. (24), Mangan et al. (25), and Veldhoen et al. (26) showed that IL-6, TGF- $\beta$, IL-1 $\beta$ and IL-23 were required for the induction of Th17 development. Despite these findings, the precise contribution of these cytokines in the development of human Th17 cells remains controversial (27). It has recently been revealed that naïve $\mathrm{CD}^{+} \mathrm{T}$ cells stimulated with TGF- $\beta 3 / \mathrm{IL}-6$, or IL$1 \beta /$ IL-6/IL-23, or TGF- $\beta 1 /$ IL-6/IL-23 in vitro can differentiate to pathogenic Th17 cells and play important roles in the occurrence of autoimmune diseases. However, TGF- $\beta 1 /$ IL-6-induced Th17 cells with suppressive function named by non-pathogenic or regulatory Th17 cells $(28,29)$.

In fact, the extremely dynamic process of Th17 subset differentiation shares an overlapping developmental axis with Th22, inducible regulatory $\mathrm{T}$ (iTreg) cells, and Th1 cells, which displays in an intermediary subsets of cells that display shared expression of lineage-specific transcription factors and cytokines (30). 
Gut-associated lymphoid tissue (GALT) is a highly specialized region of the intestine where the differentiation of activated $\mathrm{CD}^{+} \mathrm{T}$ cells takes place, and provides additional layers of complexity towards modulating Th17 plasticity (30).

It was reported at least four major pathways and/or factors which contribute to Th17 plasticity (figure 2). The pro- and anti-inflammatory cytokine milieu directs $\mathrm{CD}^{+} \mathrm{T}$ cell development and modulates plasticity via the activation of specific signaling molecules and multiple transcription factors (30). Furthermore, it has emerged that immunoregulatory microRNA (miRNA) plays a critical role in controlling gene expression, thus influencing $\mathrm{T}$ cell fate and plasticity. It was reported that transcription factor aryl hydrocarbon receptor (AhR) and its physiological and environmental ligands alongside histone methylation and epigenetic modifications may influence $\mathrm{T}$ cell plasticity (30).

\section{Th17 deficiency}

Th17 deficiency due to defects in frequency and/or function of Th17 cells leads to unusual susceptibility to C. albicans infections (31). Some conditions that impair Th17 development and function in human are monogenic syndromes that prone patients to fungal infection including CMC (table I). The most known disorders are Hyper IgE syndrome (HIES) with auto-

Figure 2 - Development and plasticity of Th17 cells with other helper T subsets.

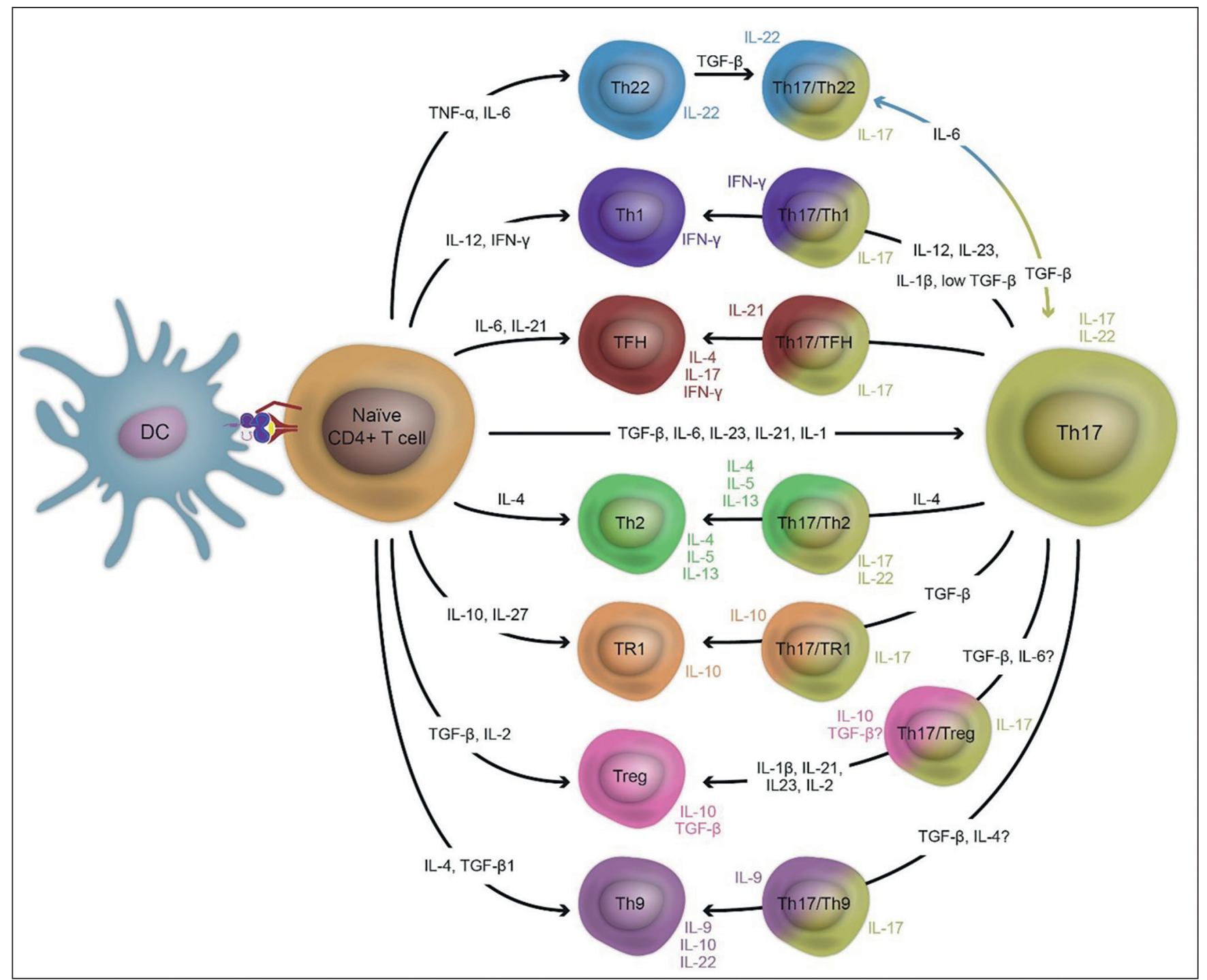


somal dominant and autosomal recessive inheritance, gain of function (GOF) mutation in STAT1, and APS-1/APECED syndrome (figure 3) (32, 33).

\section{Monogenic defects leading to Th17 deficiency}

\section{Mutations in STAT1}

A transcription factor called the signal transducer and activator of transcription 1 (STAT1) in humans is encoded by the STAT1 gene that regulates multiple biological downstream processes in a variety of cytokine receptors in many cell types (34). STAT1 mutations can be gain of function or loss of function (LOF) both of which can cause different phenotypes and symptoms. Recurring common infections are frequent in both GOF and LOF mutations. STAT1 GOF mutations, which account for approximately one-half of patients with $\mathrm{CMC}$, was originally identified in a Ukrainian patient with CMC using whole-exome sequencing (35). Subsequently, mutations affecting STAT1's coiled-coil do-

Figure 3 - Monogenic diseases that impair Th17 development or function against fungal pathogen. IL-1 $\beta, I L-6, I L-23, T N F-\alpha$ and TGF- $\beta$ are secreted after activation of antigen-presenting cells by fungal antigens.

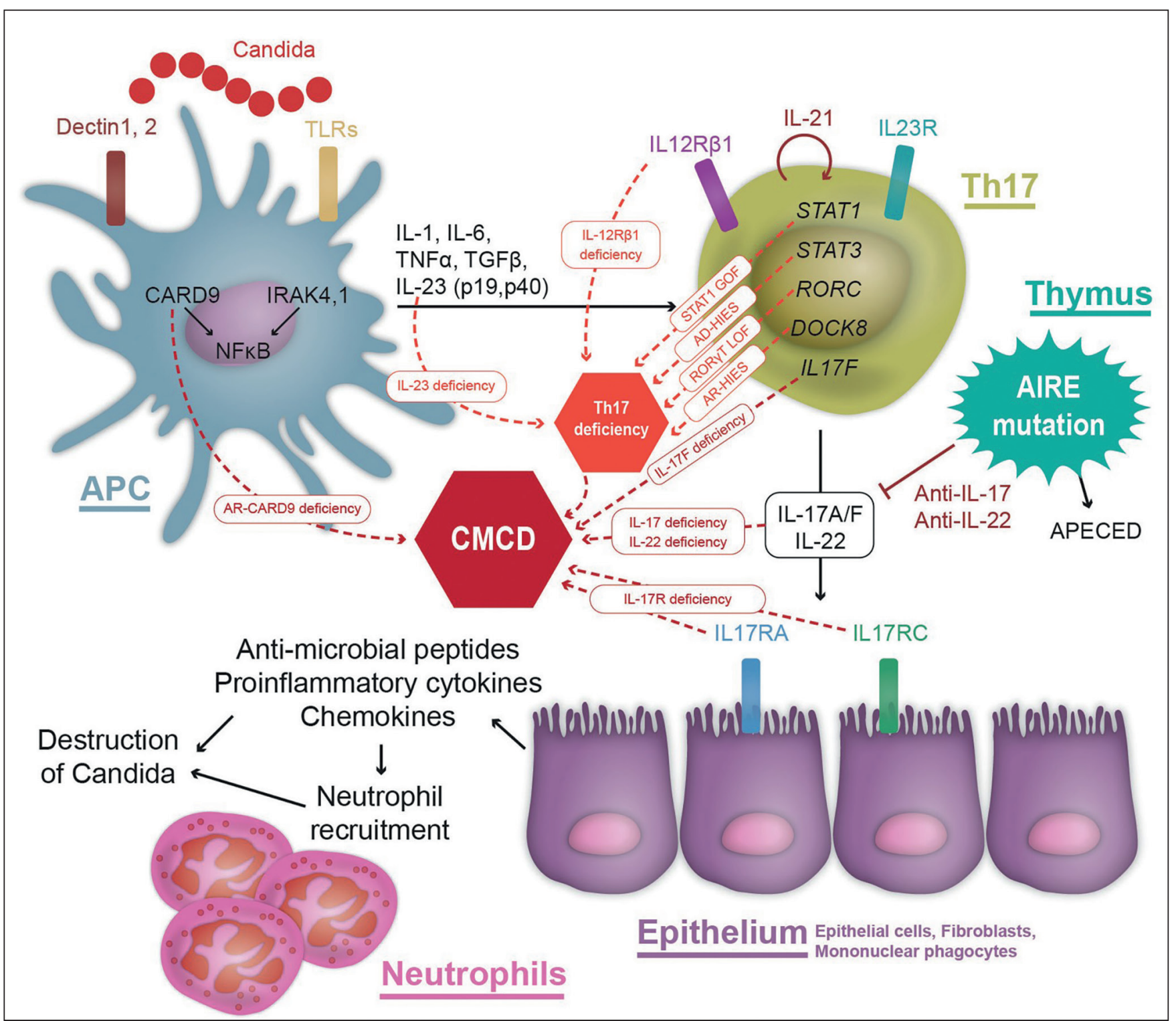


main (CCD) and DNA-binding domain (DBD) were described in a significant number of patients belonging to other kindreds (35). GOF mutations of STAT1 have been shown to be the most common etiology of CMC over the past few years (35). Heterozygous GOF mutations in STAT1 have been associated with a diverse phenotype encompassing CMC, autoimmune disease (such autoimmune hepatitis), lymphadenopathy, early-onset rosacea, and various cancers (Hodgkin Lymphoma, oesophageal cancer or squamous cell carcinoma) $(36,37)$.

Among $\mathrm{CD}^{+}$subpopulation, IL- $17^{+} \mathrm{T}$ cells impaired development resulting from $\mathrm{CD}^{+} \mathrm{T}$ cells were demonstrated in patients with CMC (35). These GOF mutations result in increased phosphorylation, DNA binding, transactivation, and interaction with protein inhibitor of activated STAT (PIAS), and decreased dephosphorylation of STAT1 (35). Thus, in patients with CMC, the poor development of IL-17+ $\mathrm{T}$ cells may be involved with increased responses to IFN- $\gamma$, IFN- $\alpha / \beta$ and IL-27, which are STAT1-dependent repressors of IL-17-producing cells (35). Low-

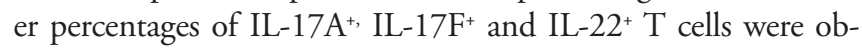
served in patients with GOF STAT1 mutations, as well as a lower production of IL-6, IL-17A and IL-22 than those of healthy control subjects, while a high level of IL-4 was reported (38). Moreover, many patients with GOF STAT1 mutations that develop autoimmune disorders exhibit improved type 1 IFN cellular immune responses, which could explain the link between STAT1 hyperactivity and the development of autoimmunity (39).

STAT1 GOF mutation that is characterized as heterozygous dominant may bring about stronger cellular immune responses towards STAT1-dependent IL-17 inhibitors (IFN- $\alpha / \beta$, IFN- $\gamma$ and IL-27) (35). This is responsible for the abnormal IL-17+ ${ }^{+}$-cell development, impacting on IL-17 $7^{+} \mathrm{T}$ cell-mediated responses that are often antifungal in effect. The establishment of safe and effective treatments based on a precise understanding of the molecular mechanisms of this disorder is required to improve patient care (40).

\section{Mutations in STAT3}

STAT3 is a transcription factor involved in signalling for a variety of cytokines, hormones and growth factors (41). The STAT3 gene plays an important role in maturation, differentiation, and function of T and B lymphocytes (42). Sequel to STAT3 involvement in Th17 cell differentiation, the stimulation of $\mathrm{CD}^{+}$ $\mathrm{T}$ cells toward the follicular helper $\mathrm{T}$ cell lineage may also depend on STAT3 (43). Naïve T cells differentiation towards Th17 cells lineage is a very complex process which is yet to be completely understood. STAT3 which is activated by IL-6 and IL-21, can play an essential role in the development of Th17 cells in humans (44). Moreover, STAT3 activation induces expression of ROR $\gamma \mathrm{t}$ which plays an important role in Th17 function (27).

Two independent groups in 2007 reported that mutation in STAT3 as a major cause of sporadic HIES and autosomal dom- inant (AD) (45). Mutations were limited in either the DBD or the Src2 homology (SH2) domains of the STAT3 gene and were later described throughout the gene in other different populations (46). Immunologic defects reported include severe increase in serum IgE levels, eosinophilia, neutrophil chemotactic defect (47), abnormal cytokine production, and abnormal antibody responses to bacteriophage $\Phi \mathrm{X} 174$ (48). As a result, patients with AD-HIES have abnormal susceptibility to a narrow spectrum of infections, such as Streptococcus aureus, and C. albicans while this may be a likely explanation for the susceptibility of these patients to CMC. Patients with dominant negative STAT3 mutations in patients present with a decreased number of central memory $\mathrm{CD}^{+}$and $\mathrm{CD} 8{ }^{+}$lymphocytes and an increased number of naïve $\mathrm{T}$ cells (49). Due to this memory $\mathrm{T}$ cell defect; these patients are predisposed to develop varicella zoster virus reactivation and prolonged Epstein-Barr virus viremia (49). Recent advances in our understanding of genetic aetiology of STAT3 mutations have established the essential role of STAT3 in Th17 cell differentiation (32). STAT3 deficient patients were assessed by Ma $e t$ al. for any possible potential defects in Th17 cells development (32). Examination of premature $\mathrm{T}$ cell produced from the total $\mathrm{CD}^{+} \mathrm{T}$ cells of patients showed significant decrease in IL-17 ${ }^{+}$ $\mathrm{T}$ cells and following stimulation of these $\mathrm{CD}^{+} \mathrm{T}$ cells resulted in the absence of IL-17 production. Additionally, IL-17 production was impaired in both groups of HIES patients, although the impairment was more severe in patients with STAT3 mutations. Thus, defective Th17 differentiation occurs by a different mechanism in AR-HIES versus AD-HIES due to STAT3 mutations. Furthermore, the production of another Th17-derived cytokine, IL-22, involved in epithelial and mucosal immunity, was also absent from $\mathrm{CD}^{+}{ }^{+} \mathrm{T}$ cells. Consistent with the vital role of STAT3 in $\mathrm{ROR} \gamma \mathrm{t}$ expression, the sorted naïve $\mathrm{CD}^{+} \mathrm{T}$ cells from the patients failed to differentiate when stimulated with anti-CD2, anti-CD3 and anti-CD28 into Th17 T cells following the presence of IL-1 $\beta$ with either IL-23 or IL-6, confirming that STAT3 is a requirement for the differentiation of Th17 cells. Furthermore, cells obtained from STAT3 deficient patients and were stimulated overnight with $S$. aureus or $C$. albicans failed to generate memory CD4 $4^{+} \mathrm{IL}-17^{+} \mathrm{T}$ cells (50). NKT-17, Tc17 and T $\gamma \delta-17$ are also sources of Th17 cytokines (51). However, CMC disease prevention do not require the production of Th17 cytokines or the production of insufficient quantities of Th17 cytokines in AD-HIES STAT3 mutation (8). This proposes the probability of STAT3 being involved in the production of Th17 cytokine in $\gamma \delta-17$ cells, Tc17 cells, and NKT-17 cells (8).

\section{Mutations in DOCK8}

DOCK8 is a member of DOCK180-related family of guanine nucleotide exchange factors (GEFs) that promotes the activity of Rho GTPases such as Rac and Cdc42 and are involved in variety 
of cellular processes including cell migration, differentiation, and cell-cell interactions $(52,53)$. DOCK8 mediates both innate and adaptive responses to ensure sufficient immune response in that it is required for lymphocyte survival, migration, and immune synapse formation (53). Consequently, its absence results in poor pathogen control (53). Though, modern advances have pointed to an essential role of DOCK8 in regulating the signal transduction events that control transcriptional activity, cytokine production and functional polarization of immune cells (54). Biallelic mutations in the DOCK8 cause progressive combined immunodeficiency (CID) characterized by susceptibility to atopic diseases, unusual susceptibility to candidal dermatitis, recurrent respiratory infections, autoimmunity and malignancy (55-58). Recent study by Haskologlu et al. (55) have reported frequent clinical manifestations in subjects with DOCK8 mutations include atopic dermatitis $(90 \%)$, recurrent respiratory tract infections (85\%), and food allergy (70\%). Failure to thrive (65\%), liver problems (60\%), bronchiectasis $(55 \%)$, chronic diarrhea $(50 \%)$, and autism spectrum disorders $(15 \%)$ were strange findings. Elevated $\mathrm{IgE}$ level (100\%) and eosinophilia (85\%), low IgM level (75\%), and decreased $\mathrm{CD}^{+} \mathrm{T}(50 \%)$ and $\mathrm{CD}^{+}{ }^{+} \mathrm{T}(55 \%)$ cell count were prominent laboratory findings. The study also revealed that, stimulation with anti-CD3 and anti-CD28 in DOCK8 deficient patients leads to $\mathrm{T}$ cell lymphopenia accompanied by poor $\mathrm{T}$ cell proliferation, and exhibit decreased Th17 and memory B cells (59). A cohort study of AR-HIES patients revealed deficiencies in the differentiation of Th17 cells (60). RORyt expression, which is critical for Th17 differentiation, was significantly reduced in peripheral $T$ cells of patients with AR-HIES. Interestingly, in vitro induction of ROR $\gamma t$ expression by naïve $T$ cells was intact. Therefore, it was suggested that initial steps of Th17 differentiation are intact in the AR-HIES patients, but subsequent steps of differentiation are impaired. It is likely that impaired Th17 differentiation and IL-17 production contributes to the susceptibility of AR-HIES patients with DOCK8 mutations to candidal dermatitis. In a more comprehensive study of functional impairment of T cell in DOCK8 deficient patients, it has been shown that the establishment of candidal infection is a result of susceptibility to viral infections. Tangye et al. (61) observed that DOCK8-deficient memory CD4 $4^{+} \mathrm{T}$ cells were partial toward a Th2 cell at the expense of Th1 and Th17 cells. Further studies into the clinical features of DOCK8-deficient $\mathrm{CD}^{+} \mathrm{T}$ cells concluded that the Th2 bias is likely to contribute to atopic disease, whereas defects in Th1 and Th17 cells compromise antiviral and antifungal immunity, respectively.

\section{Mutations in AIRE}

Autoimmune regulator (AIRE) gene is consisting of 14 exons coding for 545 amino acid protein (62). The thymic medullary epithelial cells mainly expressed the AIRE gene, which play a vital role in self-antigens presentation (63). In the pancreas, the AIRE gene is also expressed at low levels, lymph nodes, adrenal cortex, spleen and peripheral blood mononuclear cells. It is a nuclear transcriptional regulator protein involved in the ectopic expression of self-antigens in the thymus, which leads to the removal of self-reactive thymocytes and peripheral tolerance generation. The role of peripheral AIRE expression, which has been confirmed by mRNA analysis, is yet to be cleared. AIRE deficiency leads to the escape and extra-thymic spreading of autoreactive $T$ cell clones that underlie the onset of autoimmune attack against several tissue-specific self-antigens (64). Worldwide, more than 100 different mutations in this gene have been reported till date, both homogeneous and heterogeneous $(6,65)$.

Autoimmune polyendocrine syndrome 1 (APS-1) or autoimmune polyendocrinopathy candidiasis ectodermal dystrophy (APECED), which is an autosomal recessive disease as a result of mutations in the AIRE gene, characterized by CMC, adrenal insufficiency and hypoparathyroidism (65). The disease commonly begins in childhood, with the sequential onset of manifestations beginning with CMC at around five years of age, followed by hypoparathyroidism and then by adrenal insufficiency (33). Other endocrine and non-endocrine components, such as hypothyroidism, autoimmune hepatitis, type 1 diabetes, gastro-intestinal dysfunction, hypergonadotropic hypogonadism, asplenia, and various ectodermal abnormalities (nail dystrophy, intestinal keratitis, vitiligo, alopecia, and dental enamel hypoplasia) may also occur with different prevalence (66).

AIRE deficiency has been shown to enhance the differentiation of into Th1, Th17, and follicular helper $\mathrm{T}$ (Tfh) cells, while reducing the differentiation of Th2 cells and Tregs (67). These information propose that AIRE may play a role in inducing Th17 and Th1 differentiation by upregulating cytokine expression in DCs (68). Also, the autoantibodies of patients distinguish not only multiple organ-specific targets, but also most Th17 cell-associated cytokines, many type I interferons (IFNs) with neutralizing biological actions in vitro (69). These anti-cytokine autoantibodies are extremely disease-specific even though they have been found only in patients with thymomas, tumours of thymic epithelial cells that fail to express AIRE. Furthermore, autoantibodies against Th17 cell-associated cytokines associate with CMC in both syndromes (70).

The accurate diagnosis of APS- 1 requires that at least two of these three major components or only one if a sibling has already been diagnosed with the disease (6). Thus, to examine if the $\mathrm{T}$ helper reactivity to Candida albicans and other stimuli was altered, PBMCs from APS-1 patients were isolated and matched with healthy controls. In APS-1 patients, the Th17 pathway was upregulated in response to $C$. albicans, whereas there is reduction in the secretion of IL-22 (71). From the sera of APS-1 patients, autoantibodies against IL-17A, IL-17F and IL-22 were detected by immunoprecipitation. Furthermore, AIRE-deficient mice were much more vulnerable than AIRE $(+/+)$ mice to mucosal candidiasis and C. albicans induced increased responses of Th17- and Th1-cell in AIRE-deficient 
mice. Hence, in APS-1 patients, an excessive IL-17A reactivity towards C. albicans was observed and AIRE-deficient mice (71).

\section{Mutations in IL12Rß1 and IL-12p40}

IL-12 and IL-23 are heterodimeric cytokines composing of a subunit of p35 and p19, respectively, while both contain the same p40 subunit (72). The heterodimer, p19-p40, of IL-23 binds to IL-12R $\beta 1$ and make use of its receptor complex comprising of IL-12R $\beta 1$ and IL-23R on NK cells and T cells (72). IL-23 is a crucial cytokine that maintains Th17 cells expansion following differentiation from naive $\mathrm{CD}^{+}{ }^{+}$cells as a result of exposure to IL-1 $\beta$, IL-6, and IL-21 (44).

IL-12 and IL-23 receptor b1 chain deficiency and IL-12p40 deficiency are two autosomal recessive forms of Mendelian Susceptibility to mycobacterial disease (MSMD) following moderate phenotypes with particular susceptibility to Salmonella infection (73). Predominantly, intracellular pathogens were found to be responsible for a wide range of infections as result of the functional impairment of IL-12R $\beta 1$ (74). In patients with IL-12R $\beta 1$ mutation, NK cells and T cells may not be able to respond to IL-12 or IL-23, causing the frequent development of CMC in these patients (75). Patients deficient in IL-12p40 subunit joint by IL-12 and IL-23 can also be susceptible to CMC due to weakened maintenance of Th17 cells at mucosal surfaces (74). In a study performed by Prando et al. CMC was observed in $3(6.7 \%)$ of 49 patients with autosomal recessive IL12p40 deficiency (76). One patient had invasive candidiasis and two presented with oral thrush. It was reported that $23 \%$ of patients with IL-12R $\beta 1$ deficiency presented with clinical features of candidiasis (74). Most candidiasis episodes are mucocutaneous, oropharyngeal candidiasis, esophageal, cutaneous, or genital. In addition to mucosal or cutaneous fungal diseases, some reports of invasive candidiasis were also presented (74). The basis of immunity resulting to lower incidence of CMC in patients with IL-12p40 deficiency compared to patients with IL-12R $\beta 1$ deficiency has not been completely understood. Both IL-12R $\beta 1$ and IL-12p40-deficient patients with reduced IL-23 signaling, demonstrate a reduced population of Th17 cells. The reduction in the Th17 population in these patients is not as severe as in STAT3-deficient patients. This suggest that IL-23 is vital for the development of Th17 cells, maintenance, or both, but some redundancy might still exist to permit reduced development of Th17 cells. Since $C$. albicans infection is uncommon in IL-12R $\beta 1$ and IL-12p40-deficient patients, it may be due to the bulk of these patients retaining an adequate Th17 function to prevent susceptibility to CMC. In addition, IL-12 has

Table I - Monogenic diseases that impair Th17 development or function.

\begin{tabular}{|c|c|c|}
\hline Genetic defect & Phenotypic characteristics & References \\
\hline STAT1 (GOF) & $\begin{array}{l}\text { Chronic mucocutaneous candidiasis, Autoimmune hepatitis, Autoimmune } \\
\text { hemolysis, hypothyroidism, lymphadenopathy, Early-onset rosacea, } \\
\text { Cancers like Hodgkin's lymphoma, Oesophageal cancer or Squamous cells } \\
\text { carcinoma, Pneumonia, P. jirovecii pneumonia, CMV }\end{array}$ & $\begin{array}{l}(36) \\
(37)\end{array}$ \\
\hline STAT3 & $\begin{array}{l}\text { AD-HIES, elevated IgE, S. aureus abscesses, Pneumonia, Pneumatocele } \\
\text { formation, Candidiasis }\end{array}$ & $\begin{array}{l}(90) \\
(91)\end{array}$ \\
\hline AIRE & $\begin{array}{c}\text { Chronic mucocutaneous candidiasis, Adrenal insufficiency, } \\
\text { Hypoparathyroidism, Autoimmune diseases, Hypogonadism, Asplenia, } \\
\text { Various ectodermal abnormalities, Splenomegaly, Arthralgia, Autoimmune } \\
\text { polyendocrine syndrome } 1\end{array}$ & $\begin{array}{l}(33) \\
(66) \\
(95)\end{array}$ \\
\hline IL-22 & Multiple Sclerosis & $(99)$ \\
\hline RORC & $\begin{array}{l}\text { Oxazolone-induced colitis in mice } \\
\text { Pancreatic cancer progression and lymph node invasion, Lung cancer, } \\
\text { Breast cancer, Neuroendocrine prostate cancer }\end{array}$ & $\begin{array}{l}(100) \\
(101) \\
(101)\end{array}$ \\
\hline IL-12Rß1 \& IL-12p40 deficiency & Multiple Sclerosis, Mendelian Susceptibility to Mycobacterial Diseases & $\begin{array}{l}(8) \\
(77)\end{array}$ \\
\hline
\end{tabular}


been shown to play a significant role in immunity against candida (77). Last but not least, patients with IL-12p40 and IL-12R $\beta 1$ deficiencies are evidently deficient in IL-12 responses, which account for their mycobacterial susceptibility (8).

\section{Diagnosis of Th17 deficiency}

Patients, who exclusively present with recurrent $S$. aureus and Candida species infection and a high IgE serum level, should be considered for the diagnosis of Th17 deficiency. Laboratory anomalies in these patients may include defective cutaneous or in vitro T-cell Candida species response. Patients with Th17 deficiency associated with STAT1, IL17RA, IL17F and CARD9 mutations will not have other defects in humoral or cellular immunodeficiencies $(78,79)$. Laboratory testing might reveal impaired in vitro lymphocyte proliferation and cytokine secretion in response to Candida species, while delayed-type hypersensitivity test results to Candida species might be normal. In patients with mutations in CARD9 and STAT1, a decrease in Th17 cell counts has been observed, although its frequency is normal in those with mutations in IL17RA and IL17F (80, 81). Immunologic abnormalities are variable in patients with DOCK8 mutations, but CID may be considered when including both cellular and humoral immune deficiencies. In a few patients with DOCK8 deficiency, TREC numbers have been found to be low (82). Based on Th17 cells surface (CD4, CCR4, and CCR6) and intracellular (IL-17 and RORC) markers, flow cytometric analysis is a valuable diagnostic tool to assess the Th17 cells frequency and function in suspect patients (83). In a study Meshaal et al. proposed diagnosis of DOCK8 deficiency using flow cytometry biomarkers (84). In their study, profound defects in Th17 cells and Tregs were observed in all patients with impaired STAT3 phosphorylation, indicating that DOCK8 plays a pivotal role in the STAT3 signaling pathway. These findings along with detecting diminished memory B cell numbers and defective DOCK8 expression by flow cytometry can confirm the diagnosis.

Serum levels of IL-17A and IL-22 and anti-IL-17A, anti-IL-17F, and anti-IL-22 autoantibodies can be measured by using commercially available enzyme-linked immunosorbent assay (ELISA) and chemilumiscence assays (CLIA). However, confirmation of Th17 deficiency syndromes by genetic screening of suspected patients with molecular new techniques such next generation sequencing (NGS) or evaluation of responsible genes (STAT1, STAT3, DOCK8, AIRE, $R O R C, I L-17, I L-17 R$, and $I L-22)$ by Sanger sequencing in patients with related phenotype should not be neglected (78).

\section{Therapeutic approach in Th17 deficiency}

Treatments for monogenic patients with Th17 deficiency involve preventing and treating infections, boosting the immune system, and treating the underlying cause of the immune problem. Prolonged treatment with antifungal agents might be prerequisite, depending on the extent of Candida species infection. Eczem- atous dermatitis requires rigorous topical therapy with steroids and a moisturizing cream. Topical application of calcineurin inhibitors such as Pimecrolimus and Tacrolimus may also be used for controlling eczematous lesions (78). In patients with prominent autoimmune complication such as APECED syndrome, immunomodulating drugs can also subdue clinical manifestations, but their immunosuppressive actions should be carefully monitored to avoid severe complications caused by underlying host defense abnormalities. The primary treatments for affected patients with APECED syndrome include antifungals to treat mucocutaneous candidiasis and hormone replacement for endocrinopathies (85). It was revealed that early HSCT is associated with better outcomes in some type of patients with Th17 deficiency. Successful HSCT for patients with DOCK8 mutation has been reported in several cases. HSCT has been shown to cure nearly all clinical and laboratory manifestations by reconstituting the normal function of the immune system $(86,87)$. In a cohort study by Haskologlu et al., HSCT led to a marked improvement in atopic dermatitis and food allergies, along with decreasing infection frequency. The overall survival was $91 \%$ in HSCT-received patients (55). Recently, a study reported HSCT in cases with STAT3 mutation have had successful outcomes (88). However, in one early reported case of HSCT for STAT3 mutation, the clinical manifestations reappeared (89).

\section{Conclusions}

Th17 deficiency is an abnormality that should be considered in the diagnosis of patients who demonstrate recurrent infections and susceptibility to fungal infections. When diagnosing such patients, care should be taken in assessing the clinical symptoms, as well as the possibility of identifying the responsible genetic defect associated with the disease, and these may, eventually, result to an effective and accurate management or even treatment of the disease.

\section{Fundings}

This work was supported by the vice chancellor for research, Alborz University of Medical Sciences, under Grant No. 1398-1-99-3613.

\section{Conflict of interests}

The authors declare that they have no conflict of interests.

\section{References}

1. Korn T, Bettelli E, Oukka M, Kuchroo VK. IL-17 and Th17 Cells. Annu Rev Immunol 2009;27:485-517.

2. Mengesha BG, Conti HR. The Role of IL-17 in Protection against Mucosal Candida Infections. J Fungi (Basel) 2017;3(4):52.

3. Hernandez-Santos N, Gaffen SL. Th17 cells in immunity to Candida albicans. Cell Host Microbe 2012;11(5):425-35.

4. Ishigame H, Kakuta S, Nagai T, et al. Differential roles of interleukin-17A and $-17 \mathrm{~F}$ in host defense against mucoepithelial bacterial infection and allergic responses. Immunity 2009;30(1):108-19. 
5. Tamaura M, Satoh-Takayama N, Tsumura M, et al. Human gainof-function STAT1 mutation disturbs IL-17 immunity in mice. Int Immunol 2020;32(4):259-72.

6. Humbert L, Cornu M, Proust-Lemoine E, et al. Chronic Mucocutaneous Candidiasis in Autoimmune Polyendocrine Syndrome Type 1. Front Immunol 2018;9:2570.

7. Bousfiha A, Jeddane L, Picard C, et al. Human Inborn Errors of Immunity: 2019 Update of the IUIS Phenotypical Classification. J Clin Immunol 2020;40(1):66-81.

8. McDonald DR. TH17 deficiency in human disease. J Allergy Clin Immunol 2012;129(6):1429-35; quiz 36-7.

9. Shabgah AG, Fattahi E, Shahneh FZ. Interleukin-17 in human inflammatory diseases. Postepy Dermatol Alergol 2014;31(4):256-61.

10. Fu B, Tian Z, Wei H. TH17 cells in human recurrent pregnancy loss and pre-eclampsia. Cell Mol Immunol 2014;11(6):564-70.

11. Khader SA, Bell GK, Pearl JE, et al. IL-23 and IL-17 in the establishment of protective pulmonary CD4+ T cell responses after vaccination and during Mycobacterium tuberculosis challenge. Nat Immunol 2007;8(4):369-77.

12. Umemura M, Yahagi A, Hamada S, et al. IL-17-mediated regulation of innate and acquired immune response against pulmonary Mycobacterium bovis bacille Calmette-Guerin infection. J Immunol 2007;178(6):3786-96.

13. Liang SC, Tan XY, Luxenberg DP, et al. Interleukin (IL)-22 and IL17 are coexpressed by Th17 cells and cooperatively enhance expression of antimicrobial peptides. J Exp Med 2006;203(10):2271-9.

14. Chung Y, Yang X, Chang SH, Ma L, Tian Q, Dong C. Expression and regulation of IL-22 in the IL-17-producing CD4+ T lymphocytes. Cell Res 2006;16(11):902-7.

15. Scriba TJ, Kalsdorf B, Abrahams DA, et al. Distinct, specific IL-17- and IL-22-producing CD4+ T cell subsets contribute to the human anti-mycobacterial immune response. J Immunol 2008;180(3):1962-70.

16. Happel KI, Dubin PJ, Zheng M, Ghilardi N, Lockhart C, Quinton LJ, et al. Divergent roles of IL-23 and IL-12 in host defense against Klebsiella pneumoniae. J Exp Med 2005;202(6):761-9.

17. Caruso R, Fina D, Paoluzi OA, et al. IL-23-mediated regulation of IL-17 production in Helicobacter pylori-infected gastric mucosa. Eur J Immunol 2008;38(2):470-8.

18. Rudner XL, Happel KI, Young EA, Shellito JE. Interleukin-23 (IL23)-IL-17 cytokine axis in murine Pneumocystis carinii infection. Infect Immun 2007;75(6):3055-61.

19. Kleinschek MA, Muller U, Brodie SJ, et al. IL-23 enhances the inflammatory cell response in Cryptococcus neoformans infection and induces a cytokine pattern distinct from IL-12. J Immunol 2006;176(2):1098-106.

20. Acosta-Rodriguez EV, Rivino L, Geginat J, et al. Surface phenotype and antigenic specificity of human interleukin 17-producing T helper memory cells. Nat Immunol 2007;8(6):639-46.

21. Zelante T, De Luca A, Bonifazi P, et al. IL-23 and the Th17 pathway promote inflammation and impair antifungal immune resistance. Eur J Immunol 2007;37(10):2695-706.

22. De Luca A, Montagnoli C, Zelante T, et al. Functional yet balanced reactivity to Candida albicans requires TRIF, MyD88, and IDO-dependent inhibition of Rorc. J Immunol 2007;179(9):5999-6008.

23. Cosmi L, De Palma R, Santarlasci V, et al. Human interleukin 17-producing cells originate from a CD161+CD4+ T cell precursor. J Exp Med 2008;205(8):1903-16.
24. Bettelli E, Carrier Y, Gao W, et al. Reciprocal developmental pathways for the generation of pathogenic effector TH17 and regulatory T cells. Nature 2006;441(7090):235-8.

25. Mangan PR, Harrington LE, O'Quinn DB, Helms WS, Bullard $\mathrm{DC}$, Elson $\mathrm{CO}$, et al. Transforming growth factor-beta induces development of the $\mathrm{T}(\mathrm{H}) 17$ lineage. Nature 2006;441(7090):231-4.

26. Veldhoen M, Hocking RJ, Atkins CJ, Locksley RM, Stockinger B. TGFbeta in the context of an inflammatory cytokine milieu supports de novo differentiation of IL-17-producing T cells. Immunity 2006;24(2):179-89.

27. Manel N, Unutmaz D, Littman DR. The differentiation of human $\mathrm{T}(\mathrm{H})-17$ cells requires transforming growth factor-beta and induction of the nuclear receptor RORgammat. Nat Immunol 2008;9(6):641-9.

28. Wu X, Tian J, Wang S. Insight Into Non-Pathogenic Th17 Cells in Autoimmune Diseases. Front Immunol 2018;9:1112.

29. Singh B, Schwartz JA, Sandrock C, Bellemore SM, Nikoopour E. Modulation of autoimmune diseases by interleukin (IL)-17 producing regulatory $\mathrm{T}$ helper (Th17) cells. Indian J Med Res 2013;138(5):591-4.

30. Bhaumik S, Basu R. Cellular and Molecular Dynamics of Th17 Differentiation and its Developmental Plasticity in the Intestinal Immune Response. Front Immunol 2017;8:254.

31. Dhalla F, Fox H, Davenport EE, et al. Chronic mucocutaneous candidiasis: characterization of a family with STAT-1 gain-of-function and development of an ex-vivo assay for Th17 deficiency of diagnostic utility. Clin Exp Immunol 2016;184(2):216-27.

32. Ma CS, Chew GY, Simpson N, et al. Deficiency of Th17 cells in hyper IgE syndrome due to mutations in STAT3. J Exp Med 2008;205(7):1551-7.

33. Improda N, Capalbo D, Cirillo E, et al. Cutaneous vasculitis in patients with autoimmune polyendocrine syndrome type 1: report of a case and brief review of the literature. BMC Pediatr 2014; 14:272.

34. Ovadia A, Sharfe N, Hawkins C, Laughlin S, Roifman CM. Two different STAT1 gain-of-function mutations lead to diverse IFN-gamma-mediated gene expression. NPJ Genom Med 2018;3:23.

35. Soltesz B, Toth B, Shabashova N, et al. New and recurrent gainof-function STAT1 mutations in patients with chronic mucocutaneous candidiasis from Eastern and Central Europe. J Med Genet 2013;50(9):567-78.

36. Henrickson SE, Dolan JG, Forbes LR, et al. Gain-of-Function STAT1 Mutation With Familial Lymphadenopathy and Hodgkin Lymphoma. Front Pediatr 2019;7:160.

37. Sáez-de-Ocariz M, Suárez-Gutiérrez M, Migaud M, et al. Rosacea as a striking feature in family members with a STAT1 gain-of-function mutation. J Eur Acad Dermatol Venereol 2020;34(6):e265-e267.

38. Martinez-Martinez L, Martinez-Saavedra MT, Fuentes-Prior P, et al. A novel gain-of-function STAT1 mutation resulting in basal phosphorylation of STAT1 and increased distal IFN-gamma-mediated responses in chronic mucocutaneous candidiasis. Mol Immunol 2015;68(2 Pt C):597-605.

39. Di Domizio J, Cao W. Fueling autoimmunity: type I interferon in autoimmune diseases. Expert Rev Clin Immunol 2013;9(3):201-10.

40. Tamaura M, Satoh-Takayama N, Tsumura M, et al. Human Gainof-Function STAT1 Mutation disturbs IL-17 Immunity in Mice. Int Immunol 2019;32(4):259-72.

41. O’Shea JJ, Lahesmaa R, Vahedi G, Laurence A, Kanno Y. Genomic views of STAT function in CD4+ T helper cell differentiation. Nat Rev Immunol 2011;11(4):239-50. 
42. Kane A, Deenick EK, Ma CS, Cook MC, Uzel G, Tangye SG. STAT3 is a central regulator of lymphocyte differentiation and function. Curr Opin Immunol 2014;28:49-57.

43. Ma CS, Avery DT, Chan A, et al. Functional STAT3 deficiency compromises the generation of human $\mathrm{T}$ follicular helper cells. Blood 2012;119(17):3997-4008.

44. McGeachy MJ, Cua DJ. Th17 cell differentiation: the long and winding road. Immunity 2008;28(4):445-53.

45. Minegishi Y, Saito M, Tsuchiya S, et al. Dominant-negative mutations in the DNA-binding domain of STAT3 cause hyper-IgE syndrome. Nature 2007;448(7157):1058-62.

46. Chandesris MO, Melki I, Natividad A, et al. Autosomal dominant STAT3 deficiency and hyper-IgE syndrome: molecular, cellular, and clinical features from a French national survey. Medicine (Baltimore) 2012;91(4):e1-19.

47. Hill HR, Ochs HD, Quie PG, et al. Defect in neutrophil granulocyte chemotaxis in Job's syndrome of recurrent "cold" staphylococcal abscesses. Lancet 1974;2(7881):617-9.

48. Renner ED, Torgerson TR, Rylaarsdam S, et al. STAT3 mutation in the original patient with Job's syndrome. N Engl J Med 2007;357(16):1667-8.

49. Siegel AM, Heimall J, Freeman AF, et al. A critical role for STAT3 transcription factor signaling in the development and maintenance of human T cell memory. Immunity 2011;35(5):806-18.

50. Minegishi Y, Saito M, Nagasawa M, et al. Molecular explanation for the contradiction between systemic Th17 defect and localized bacterial infection in hyper-IgE syndrome. J Exp Med 2009;206(6):1291-301.

51. Cua DJ, Tato CM. Innate IL-17-producing cells: the sentinels of the immune system. Nat Rev Immunol 2010;10(7):479-89.

52. Laurin M, Cote JF. Insights into the biological functions of Dock family guanine nucleotide exchange factors. Genes Dev 2014;28(6):533-47.

53. Kearney CJ, Vervoort SJ, Ramsbottom KM, et al. DOCK8 Drives Src-Dependent NK Cell Effector Function. J Immunol 2017:ji1700751.

54. Kearney CJ, Randall KL, Oliaro J. DOCK8 regulates signal transduction events to control immunity. Cell Mol Immunol 2017;14(5):406-11.

55. Haskologlu S, Kostel Bal S, Islamoglu C, et al. Clinical, immunological features and follow up of 20 patients with dedicator of cytokinesis 8 (DOCK8) deficiency. Pediatr Allergy Immunol 2020;31(5):515-527.

56. Zhang Q, Davis JC, Lamborn IT, et al. Combined immunodeficiency associated with DOCK8 mutations. N Engl J Med 2009;361(21):2046-55.

57. Engelhardt KR, McGhee S, Winkler S, et al. Large deletions and point mutations involving the dedicator of cytokinesis 8 (DOCK8) in the autosomal-recessive form of hyper-IgE syndrome. J Allergy Clin Immunol 2009;124(6):1289-302 e4.

58. Chu EY, Freeman AF, Jing H, et al. Cutaneous manifestations of DOCK8 deficiency syndrome. Arch Derm 2012;148(1):79-84.

59. Lee WI, Huang JL, Lin SJ, et al. Clinical, immunological and genetic features in Taiwanese patients with the phenotype of hyper-immunoglobulin $\mathrm{E}$ recurrent infection syndromes (HIES). Immunobiology 2011;216(8):909-17.

60. Al Khatib S, Keles S, Garcia-Lloret M, et al. Defects along the T(H) 17 differentiation pathway underlie genetically distinct forms of the hyper IgE syndrome. J Allergy Clin Immunol 2009;124(2):342-8, 8 e1-5.
61. Tangye SG, Pillay B, Randall KL, et al. Dedicator of cytokinesis 8-deficient CD4(+) T cells are biased to a TH2 effector fate at the expense of TH1 and TH17 cells. J Allergy Clin Immunol 2017;139(3):933-49.

62. Su MA, Anderson MS. Aire: an update. Curr Opin Immunol 2004;16(6):746-52.

63. Pereira LE, Bostik P, Ansari AA. The development of mouse APECED models provides new insight into the role of AIRE in immune regulation. Clin Dev Immunol 2005;12(3):211-6.

64. Atquet V, Lienart F, Vaes M. Autoimmune polyendocrine syndrome and thrombocytosis. Acta Clin Belg 2015;70(6):457-60.

65. Zhu W, Hu Z, Liao X, et al. A new mutation site in the AIRE gene causes autoimmune polyendocrine syndrome type 1 . Immunogenetics 2017;69(10):643-51.

66. Capalbo D, Giardino G, Martino LD, et al. Genetic basis of altered central tolerance and autoimmune diseases: a lesson from AIRE mutations. Int Rev Immunol 2012;31(5):344-62.

67. Huo F, Li D, Zhao B, et al. Deficiency of autoimmune regulator impairs the immune tolerance effect of bone marrow-derived dendritic cells in mice. Autoimmunity 2018;51(1):10-7.

68. Li H, Li D, Sun J, Li Y, Yang W, Li Y. Autoimmune regulatoroverexpressing dendritic cells induce T helper 1 and T helper 17 cells by upregulating cytokine expression. Mol Med Rep 2016;13(1):565-71.

69. Karner J, Meager A, Laan M, et al. Anti-cytokine autoantibodies suggest pathogenetic links with autoimmune regulator deficiency in humans and mice. Clin Exp Immunol 2013;171(3):263-72.

70. Heikkila N, Laakso SM, Mannerstrom H, et al. Expanded CD4(+) Effector/Memory T Cell Subset in APECED Produces Predominantly Interferon Gamma. J Clin Immunol 2016;36(6):555-63.

71. Ahlgren KM, Moretti S, Lundgren BA, et al. Increased IL-17A secretion in response to Candida albicans in autoimmune polyendocrine syndrome type 1 and its animal model. Eur J Immunol 2011;41(1):235-45.

72. Watford WT, Hissong BD, Bream JH, Kanno Y, Muul L, O'Shea JJ. Signaling by IL-12 and IL-23 and the immunoregulatory roles of STAT4. Immunol Rev 2004;202:139-56.

73. Bousfiha A, Jeddane L, Picard C, et al. The 2017 IUIS Phenotypic Classification for Primary Immunodeficiencies. J Clin Immunol 2018;38(1):129-43.

74. Filipe-Santos O, Bustamante J, Chapgier A, et al. Inborn errors of IL-12/23- and IFN-gamma-mediated immunity: molecular, cellular, and clinical features. Semin Immunol 2006;18(6):347-61.

75. Ouederni M, Sanal O, Ikinciogullari A, et al. Clinical features of Candidiasis in patients with inherited interleukin 12 receptor beta1 deficiency. Clin Infect Dis 2014;58(2):204-13.

76. Prando C, Samarina A, Bustamante J, et al. Inherited IL-12p40 deficiency: genetic, immunologic, and clinical features of 49 patients from 30 kindreds. Medicine (Baltimore) 2013;92(2):109-22.

77. Moradi L, Cheraghi T, Yazdani R, et al. Mendelian susceptibility to mycobacterial disease: Clinical and immunological findings of patients suspected for IL12Rbeta1 deficiency. Allergol Immunopathol (Madr) 2019;47(5):491-8.

78. Bonilla FA, Khan DA, Ballas ZK, et al. Practice parameter for the diagnosis and management of primary immunodeficiency. J Allergy Clin Immunol 2015;136(5):1186-205.e1-78.

79. Palma-Carlos AG, Palma-Carlos ML. Chronic mucocutaneous candidiasis revisited. Allerg Immunol (Paris) 2001;33(6):229-32.

80. Puel A, Cypowyj S, Marodi L, Abel L, Picard C, Casanova JL. Inborn errors of human IL-17 immunity underlie chronic mucocutaneous candidiasis. Curr Opin Allergy Clin Immunol 2012;12(6):616-22. 
81. Liu L, Okada S, Kong XF, et al. Gain-of-function human STAT1 mutations impair IL-17 immunity and underlie chronic mucocutaneous candidiasis. J Exp Med 2011;208(8):1635-48.

82. Dasouki M, Okonkwo KC, Ray A, et al. Deficient T Cell Receptor Excision Circles (TRECs) in autosomal recessive hyper IgE syndrome caused by DOCK8 mutation: implications for pathogenesis and potential detection by newborn screening. Clin Immunol 2011;141(2):128-32.

83. Wacleche VS, Goulet JP, Gosselin A, et al. New insights into the heterogeneity of Th17 subsets contributing to HIV-1 persistence during antiretroviral therapy. Retrovirology 2016;13(1):59.

84. Meshaal SS, El Hawary RE, Eldash A, et al. Diagnosis of DOCK8 deficiency using Flow cytometry Biomarkers: an Egyptian Center experience. Clin Immunol 2018;195:36-44.

85. D’Hennezel E, Bin Dhuban K, Torgerson T, Piccirillo CA. The immunogenetics of immune dysregulation, polyendocrinopathy, enteropathy, X linked (IPEX) syndrome. J Med Genet 2012;49(5):291-302.

86. Metin A, Tavil B, Azik F, et al. Successful bone marrow transplantation for DOCK8 deficient hyper IgE syndrome. Pediatr Transplant 2012;16(4):398-9.

87. Lopes J, Teixeira D, Sousa C, Baptista A, Moreira D, Ferreira EO. Autosomal recessive hyper-IgE syndrome successfully treated with hematopoietic stem cell transplantation. Pediatr Dermatol 2019;36(5):693-6.

88. Goussetis E, Peristeri I, Kitra V, et al. Successful long-term immunologic reconstitution by allogeneic hematopoietic stem cell transplantation cures patients with autosomal dominant hyper-IgE syndrome. J Allergy Clin Immunol 2010;126(2):392-4.

89. Gennery AR, Flood TJ, Abinun M, Cant AJ. Bone marrow transplantation does not correct the hyper IgE syndrome. Bone Marrow Transplant 2000;25(12):1303-5.

90. Vogel TP, Milner JD, Cooper MA. The Ying and Yang of STAT3 in Human Disease. J Clin Immunol 2015;35(7):615-23.
91. Olbrich P, Freeman AF. STAT1 and STAT3 mutations: important lessons for clinical immunologists. Expert Rev Clin Immunol 2018;14(12):1029-41.

92. Hagl B, Spielberger BD, Thoene S, et al. Somatic alterations compromised molecular diagnosis of DOCK8 hyper-IgE syndrome caused by a novel intronic splice site mutation. Sci Rep 2018;8(1):16719.

93. Al Shekaili L, Sheikh F, Al Gazlan S, et al. Novel mutation in DOCK8-HIES with severe phenotype and successful transplantation. Clin Immunol 2017;178:39-44.

94. Krgovic D, Kokalj Vokac N, Zagorac A, Gregoric Kumperscak H. Rare structural variants in the DOCK8 gene identified in a cohort of 439 patients with neurodevelopmental disorders. Sci Rep 2018;8(1):9449.

95. King K, O'Gorman C, Gallagher S. Thyroid dysfunction in children with Down syndrome: a literature review. Ir J Med Sci 2014;183(1):1-6.

96. Martin DA, Towne JE, Kricorian G, et al. The emerging role of IL17 in the pathogenesis of psoriasis: preclinical and clinical findings. J Invest Dermatol 2013;133(1):17-26.

97. Lowes MA, Suarez-Farinas M, Krueger JG. Immunology of psoriasis. Annu Rev Immunol 2014;32:227-55.

98. Yan C, Huang WY, Boudreau J, Mayavannan A, Cheng Z, Wang J. IL-17R deletion predicts high-grade colorectal cancer and poor clinical outcomes. Int J Cancer 2019;145(2):548-58.

99. Gomez-Fernandez P, Lopez de Lapuente Portilla A, Astobiza I, et al. The Rare IL22RA2 Signal Peptide Coding Variant rs 28385692 Decreases Secretion of IL-22BP Isoform-1, -2 and -3 and Is Associated with Risk for Multiple Sclerosis. Cells 2020;9(1):175.

100. Hegazy AN, Powrie F. MICROBIOME. Microbiota RORgulates intestinal suppressor T cells. Science 2015;349(6251):929-30.

101. Lytle NK, Ferguson LP, Rajbhandari N, et al. A Multiscale Map of the Stem Cell State in Pancreatic Adenocarcinoma. Cell 2019;177(3):572-86.e22. 